\title{
Optimum Design of Chamfer Distance Transforms
}

\author{
Muhammad Akmal Butt, Student Member, IEEE, and Petros Maragos, Fellow, IEEE
}

\begin{abstract}
The distance transform has found many applications in image analysis. Chamfer distance transforms are a class of discrete algorithms that offer a good approximation to the desired Euclidean distance transform at a lower computational cost. They can also give integer-valued distances that are more suitable for several digital image processing tasks. The local distances used to compute a chamfer distance transform are selected to minimize an approximation error. In this paper, a new geometric approach is developed to find optimal local distances. This new approach is easier to visualize than the approaches found in previous work, and can be easily extended to chamfer metrics that use large neighborhoods. A new concept of critical local distances is presented which reduces the computational complexity of the chamfer distance transform without increasing the maximum approximation error.
\end{abstract}

Index Terms - Chamfer metrics, critical local distances, distance transforms.

\section{INTRODUCTION}

$\mathbf{T}$ HE DISTANCE transform has been applied in many image analysis tasks including shape description, feature detection, skeletonization, segmentation, and multiscale morphological filtering [1]-[5]. Since the computational cost of the exact Euclidean distance transform is relatively high, several fast algorithms have been developed to approximate it. Some of these algorithms also yield integer-valued distances, which have the additional advantage of making various image analysis tasks more efficient, e.g., skeletonization. A major class of such algorithms is based on chamfer metrics [3]. The term chamfer (introduced in [6]) originally referred to a sequential two-pass distance transform algorithm developed by Rosenfeld and Pfaltz [1] and later improved and generalized by Borgefors [3]. Even though this class of algorithms can also be implemented using parallel or queue-based algorithms, the word "chamfer" is retained for compatibility with previous work. Sometimes the word "weighted" is also used to refer to this class of distance transforms.

The chamfer distance transform approximates the global distance computation with repeated propagation of local distances within a small neighborhood mask. The approximation error depends upon the size of the neighborhood and the selection of the local distances. Borgefors [3] analyzed this problem by finding local distances that minimize the maximum absolute

Manuscript received October 14, 1996; revised December 4, 1997. This work was supported by the Joint Services Electronics Program under Contract DAAH-04-96-1-0161 and the National Science Foundation under Grant MIP94-21677. The associate editor coordinating the review of this manuscript and approving it for publication was Dr. Josef Bigun.

The authors are with the School of Electrical and Computer Engineering, Georgia Institute of Technology, Atlanta, GA 30332 USA (e-mail: akmal@ee.gatech.edu).

Publisher Item Identifier S 1057-7149(98)06814-6. error (MAE) around the boundary of a square of general size $(2 M) \times(2 M)$. The error was normalized by $M$, which gives unequal scale weighting to errors at different points on the boundary of the square and hence different angles. Others [9]-[11] followed this approach and extended the analysis to find the optimal local distances for mean squared error (MSE). A related problem of estimating discrete lines was analyzed in [12]. Verwer [13] found optimal distances under both the MAE and MSE by minimizing the error along the boundary of a unit circle thus giving equal emphasis to the errors at different angles.

In this paper, we develop a geometric approach to find optimal local distances that has certain new viewpoints and novel aspects compared with previous approaches. These new viewpoints are motivated by the major application of multiscale morphological filtering. Since the distance transform is a compact representation of multiscale morphological dilations and erosions, our objective is to find local distances for chamfer distance transform that give the best approximations of multiscale dilations/erosions by disk structuring elements. In contrast to a previous approach [13], which compared the chamfer distance and the Euclidean distance at the same points along a unit circle, we compare the distances along the boundaries of the two balls induced by the two metrics at the same scale. (The ball induced by the chamfer metric is a polygon, whereas the Euclidean ball is a disk.) Our approach is easier to visualize geometrically, shows clearly the dependence of the approximation error on individual local distances, yields simpler error expressions used to find optimal local distances under various error minimization criteria, and leads to a new concept of critical local distances that offers reduced computation without increasing the maximum error.

\section{PRELIMINARIES}

Given a binary image $f(x, y)$, let the planar set $F=$ $\{(x, y): f(x, y)=1\}$ represent its foreground or feature set and let $F^{c}$ represent its the background. The distance transform of $F$ is defined as

$$
D_{p}(F)(x, y) \equiv \inf \left\{\|(x-u, y-v)\|_{p}:(u, v) \in F^{c}\right\}
$$

where $\|\cdot\|_{p}$ is the distance under the $\ell^{p}$ norm, $p=$ $1,2,3, \cdots, \infty$. Thus, $D_{p}(F)$ is a gray-level image with values at each pixel representing its distance to the nearest pixel of $F^{c}$. Thresholding this distance transform at various levels $r>0$ yields the multiscale morphological erosions $\ominus$ of $F$ by the balls $r B_{p} \equiv\left\{(x, y):\|(x, y)\|_{p} \leq r\right\}$ of size $r$ induced by the $\ell^{p}$ norm, i.e., $F \ominus r B_{p}=\left\{(x, y): D_{p}(F)(x, y) \geq r\right\}$. Multiscale dilations of $F$ can be obtained from the distance transform of $F^{c}$. Among the norms used, the Euclidean norm 


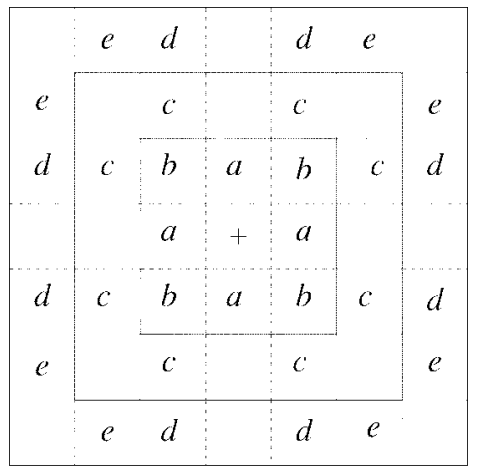

Fig. 1. Local distances for $3 \times 3,5 \times 5$, and $7 \times 7$ neighborhoods. The unmarked pixels are not used.

(where $p=2$ and $r B_{p}$ is a disk of radius $r$ ) is preferred as it gives isotropic distance measurements. A faster but approximate computation of the Euclidean distance transform can be achieved via chamfer distance transforms [3], which may also use integer arithmetic. These algorithms start from the background set $F^{c}$ and propagate local distances in $F$. At each step, distance is propagated in a small, usually $3 \times 3$ or $5 \times 5$-pixel, neighborhood. The distance to any pixel of $F$ is the sum of local distances of the shortest path starting from $F^{c}$ and ending at that pixel. This propagation of the local distances can be achieved with parallel, sequential, or queue-based algorithms [4]. If a $3 \times 3$ pixel neighborhood is used at each step of distance propagation, local distances of $a$ and $b$ are used for distances between axially and diagonally neighboring pixels, respectively; see Fig. 1 . This set of local distances is referred to as the chamfer metric $(a, b)$. Note that $(a, b)=(1,1)$ represents the chessboard metric, whereas $(a, b)=(1,2)$ gives the city-block metric [1].

\section{Optimal Local Distances for $3 \times 3$ Neighborhood}

\section{A. Error Analysis}

For the chamfer metric $(a, b)$ with regularity constraints [8] $a<b<2 a$, the chamfer distance between the origin and a planar point $(x, y)$ is the chamfer norm [3]

$$
\|(x, y)\|_{(a, b)} \equiv \max (|x|,|y|) a+\min (|x|,|y|)(b-a) .
$$

Its induced ball of size $r \geq 0$ is $r B_{(a, b)} \equiv$ $\left\{(x, y):\|(x, y)\|_{a, b} \leq r\right\}$, which is a convex polygon (octagon) henceforth called the chamfer polygon of size $r$. The goodness of approximation of the disk of radius $r$ by $r B_{(a, b)}$ depends on $a$ and $b$; examples are shown in Fig. 2. Note that (2) applies both on the continuous plane $\mathbb{R}^{2}$ as well as the integer plane $\mathbb{Z}^{2}$. The discrete chamfer polygons are sampled versions of their continuous counterparts, and for large scales the difference is very small. Our analysis will be done on the continuous plane because then the error normalized by the disk radius becomes independent of scale. Further, it is only meaningful to work with the relative error because it can be bounded, whereas the absolute error increases with scale [14].

To find the approximation error, the chamfer polygon should be compared with a disk of the same scale $r$. However, since

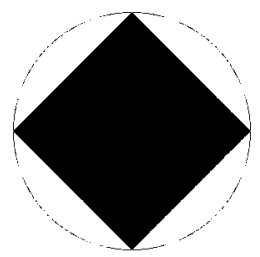

(a)

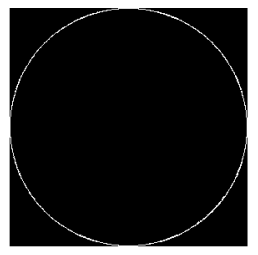

(b)

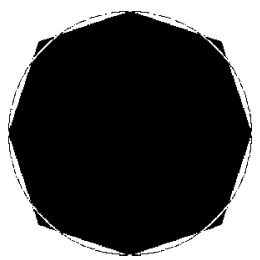

(c)
Fig. 2. Approximation to a disk of radius $r=250$ in $\mathbb{Z}^{2}$ using balls of different norms created by chamfer metrics of $3 \times 3$ neighborhood. (a) Chamfer metric $(1,2)$ or city-block norm $\|\cdot\|_{1}$. (b) Chamfer metric $(1,1)$ or chessboard norm $\|\cdot\|_{\infty}$. (c) Chamfer metric $(3,4) / 3$. (A circle of radius 250 is superimposed on all the balls.)

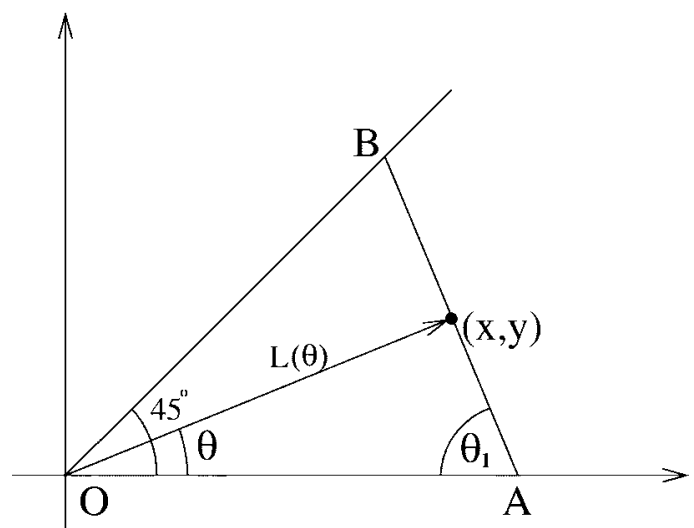

Fig. 3. Octant part of the chamfer octagon of size $r$ generated by the $3 \times$ 3 chamfer metric $(a, b)$. The end points have coordinates $A=(r / a, 0)$ and $B=(r / b, r / b)$.

the chamfer polygon is symmetric with respect to the axes and the diagonals, the analysis can be limited to the wedge-shaped planar region $\{(x, y): x \geq 0, y \geq 0$, and $x \geq y\}$. In this region, the boundary points $(x, y)$ of the chamfer polygon of size $r$ lie on the line $(b-a) y+a x=r$ which forms an edge joining two vertices $A$ and $B$, as shown in Fig. 3. The acute angle $\theta_{1}$ between the $x$-axis and this edge is equal to

$$
\theta_{1}=\tan ^{-1}\left(\frac{r / b}{r / a-r / b}\right)=\tan ^{-1}\left(\frac{a}{b-a}\right) .
$$

Applying the law of sines to the triangle formed by $O, A$, and an arbitrary point $(x, y)$ on the edge $\overline{A B}$ yields the length of the vector connecting the origin and the point $(x, y)$ on the edge of the chamfer polygon to be

$$
L(\theta)=\frac{r \sin \left(\theta_{1}\right)}{a \sin \left(\theta+\theta_{1}\right)}
$$

where $\theta$ is the angle between the vector and the $x$-axis. The approximation error is the distance difference between the point on the circle of radius $r$ and the corresponding point on the boundary of the chamfer polygon. This is equal to $r-L(\theta)$, which, when normalized by the radius $r$ yields a normalized error

$$
E(\theta) \equiv \frac{r-L(\theta)}{r}=1-\frac{\sin \left(\theta_{1}\right)}{a \sin \left(\theta+\theta_{1}\right)}, \quad 0 \leq \theta \leq \pi / 4 .
$$

The normalized error can also be obtained by comparing the unit chamfer polygon with the unit circle. Within the interval 


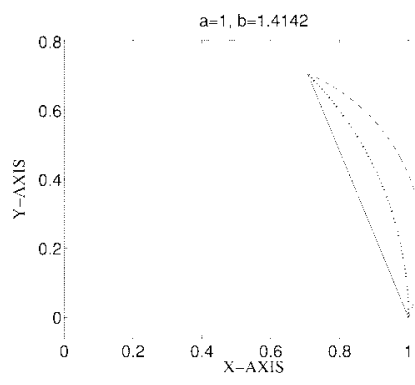

(a)

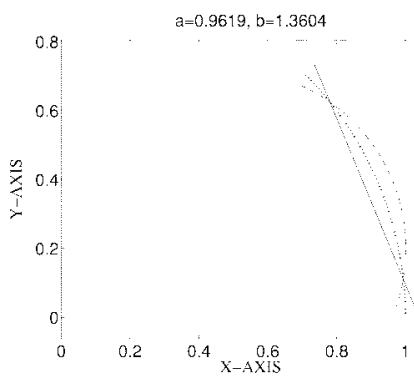

(c)

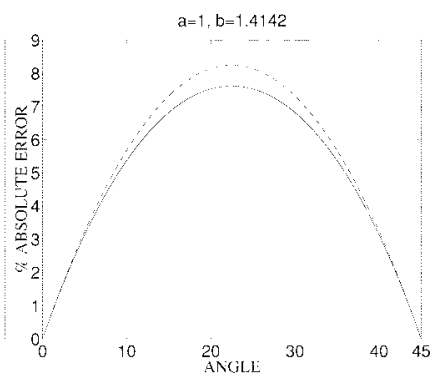

(b)

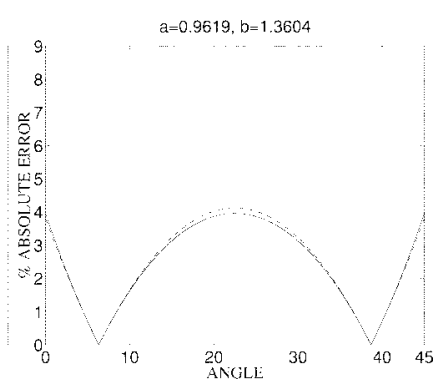

(d)

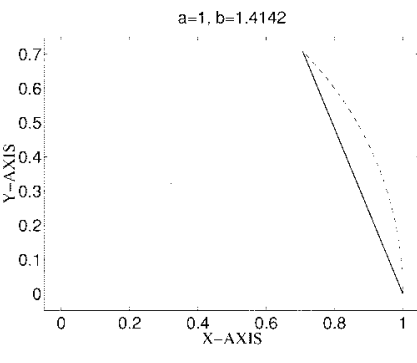

(a)

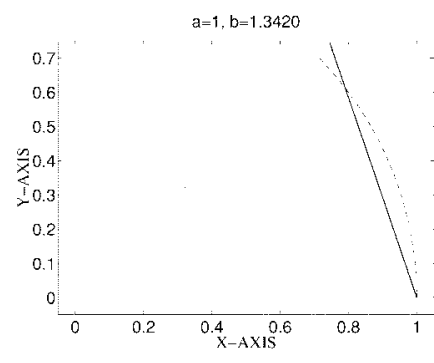

(c)

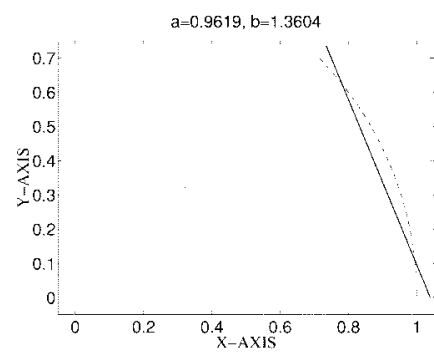

(e)

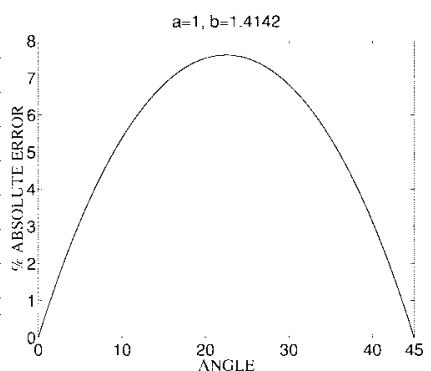

(b)

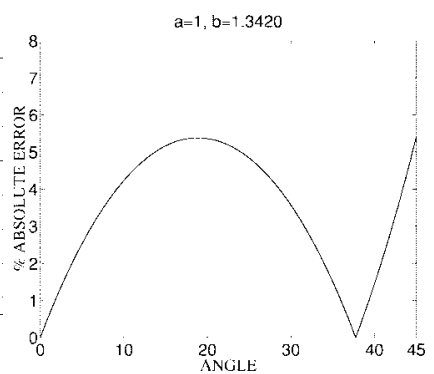

(d)

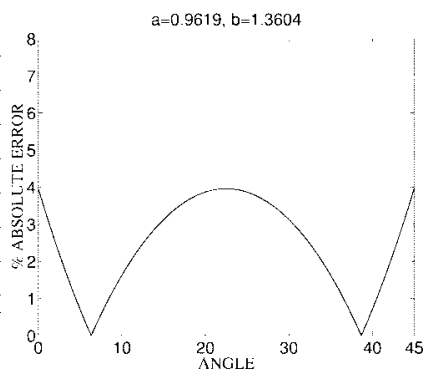

(f) $0<\theta<\pi / 4$, when $\theta=\pi / 2-\theta_{1},|E(\theta)|$ assumes its maximum value of

$\max _{0<\theta<\pi / 4}|E(\theta)||=| 1-\frac{\sin \left(\theta_{1}\right)}{a}|=| 1-\frac{1}{\sqrt{a^{2}+(b-a)^{2}}} \mid$.

However, the maximum error could also occur at $\theta=0$ or at $\theta=\pi / 4$. Hence, the complete expression for the normalized $M A E$ is

$$
\begin{aligned}
& E_{\max }(a, b) \\
& =\max \{\underbrace{\left|1-\frac{1}{a}\right|}_{\theta=0}, \underbrace{\left|1-\frac{\sqrt{2}}{b}\right|}_{\theta=\pi / 4}, \underbrace{\mid 1-\frac{1}{\sqrt{a^{2}+(b-a)^{2}} \mid}}_{0<\theta<\pi / 4}\} .
\end{aligned}
$$

\section{B. Optimal Local Distances Under the MAE Criterion}

Let us first consider the chamfer metric $(1, \sqrt{2})$ because this represents the true (Euclidean) distances between neighboring pixels. By (4), the MAE is $7.61 \%$ and occurs at $\theta=\pi / 8$; see also Fig. 5(a) and (b). The values $a=1$ and $b=\sqrt{2}$ place the vertices of the unit chamfer octagon on the unit circle. A better choice of local distances should put the vertices outside the unit circle. For example, if axial vertices are to remain on the circle $(a=1)$ and the position of the diagonal vertices,
Fig. 5. Edge geometry and normalized errors for $3 \times 3$ chamfer metrics. (a), (b) Euclidean local distances $(1, \sqrt{2})$. (c), (d) Local distances $(1,1.3420$,. (e), (f) Optimal local distances $(0.9619,1.3604)$. In (a), (c), and (e), the solid line represents the chamfer polygon, dashed line represents the circle, and dotted lines are drawn at angles of $10^{\circ}, 20^{\circ}, 30^{\circ}, 40^{\circ}$, and $45^{\circ}$.

which is controlled by the local distance $b$, is to be optimized, the expression (4) reduces to

$$
E_{\max }(1, b)=\max \left\{\left|1-\frac{\sqrt{2}}{b}\right|,\left|1-\frac{1}{\sqrt{1+(b-1)^{2}}}\right|\right\} .
$$

The diagonal corner of the normalized chamfer octagon should be moved in such a way that the error occurring at $\theta=$ $\pi / 4$ becomes equal to the maximum error occurring for $0<\theta<\pi / 4$. By equating the two error expressions in (5), we get

$$
4 b^{4}-(8+4 \sqrt{2}) b^{3}+(9+8 \sqrt{2}) b^{2}-(4+8 \sqrt{2}) b+4=0 .
$$

By solving (6) we get $b=1.342$. This gives a MAE of 5.38\%; see also Fig. 5(c) and (d).

If both $a$ and $b$ are to be optimized, they should be selected so that both axial and diagonal corners of the unit chamfer octagon are outside the unit circle by the same amount and the absolute error occurring at the corners of the normalized chamfer octagon is the same as the absolute error occurring at midpoints of the edges. This occurs when the triangle $O A B$ of 
TABLE I

Errors for Chamfer Distance Transforms Based on $3 \times 3$ Neighborhood

\begin{tabular}{c|r|r|r|l}
\hline$(a, b)$ & MAE & $\sqrt{\text { MSE }}$ & Area Diff. & Comments \\
\hline$(1,1)$ & $41.407 \%$ & $16.976 \%$ & $27.31 \%$ & Chess-board motric \\
\hline$(1,2)$ & $29.289 \%$ & $22.269 \%$ & $36.34 \%$ & City-block metric \\
\hline$(1, \sqrt{2})$ & $7.612 \%$ & $5.610 \%$ & $9.97 \%$ & Euclidean local distances \\
\hline$(3,4) / 3$ & $6.066 \%$ & $3.690 \%$ & $4.51 \%$ & A common choice \\
\hline$(1,1.3420)$ & $5.381 \%$ & $3.810 \%$ & $5.12 \%$ & Optimal for min MAE with $a=1$ \\
\hline$(0.9619,1.3604)$ & $3.957 \%$ & $2.718 \%$ & $2.70 \%$ & Optimal for min MAE \\
\hline$(70,99) / 72.77$ & $3.959 \%$ & $2.719 \%$ & $2.71 \%$ & Integer approx. for min MAE \\
\hline$(0.9489,1.3419)$ & $5.391 \%$ & $2.369 \%$ & $0 \%$ & Optimal for min MSE with Area Diff. =0 \\
\hline$(70,99) / 73.78$ & $5.400 \%$ & $2.369 \%$ & $0.01 \%$ & Integer approx. for min MSE \\
\hline
\end{tabular}

Fig. 3 is isosceles. In this case, $-E 0=E(\pi / 8)=-E(\pi / 4)$. This gives $b=\sqrt{2} a$ and $\theta_{1}=67.5^{\circ}$. Furthermore, solving $E(\pi / 8)=-E(0)$, we obtain

$$
a=\frac{1+\sin \left(67.5^{\circ}\right)}{2}=0.9619 \Rightarrow b=\sqrt{2} a=1.3604
$$

These optimal local distances give a MAE of $3.96 \%$; see also Fig. 5(e) and (f). The optimal local distances and the corresponding values of the MAE derived via our approach above are different from the values obtained by Verwer [13]. Fig. 4 further explains the difference between the two approaches.

\section{Optimal Local Distances Under the MSE Criterion}

It is possible to find optimal local distances $(a),(b)$ by minimizing the MSE. Working in the same octant planar region as for the MAE, the normalized MSE is equal to

$$
\begin{aligned}
\sigma^{2} \equiv & \frac{4}{\pi} \int_{0}^{(\pi / 4)} E^{2}(\theta) d \theta \\
= & 1+\frac{4 \sin \left(\theta_{1}\right)}{\pi a^{2}}\left(\frac{1}{\cos \left(\theta_{1}\right)+\sin \left(\theta_{1}\right)}\right. \\
& \left.\cdot-2 a \log \frac{\csc \left(\frac{\pi}{4}+\theta_{1}\right)-\cot \left(\frac{\pi}{4}+\theta_{1}\right)}{\csc \left(\theta_{1}\right)-\cot \left(\theta_{1}\right)}\right) .
\end{aligned}
$$

We have experimented with three approaches to minimize the MSE, as follows.

1) Unconstrained minimization of MSE.

2) Minimization of MSE under the zero mean error constraint $\int_{0}^{\pi / 4} E(\theta) d \theta=0$; this gives an unbiased MSE and is the same approach as in [9], [10], and [13] although our resulting optimal $(a, b)$ are different because our error function $E(\theta)$ is different.

3) Minimization of MSE under a new constraint of zero area difference, where we forced the area difference between the chamfer polygon and the disk at same scale to be zero.
For the $3 \times 3$ neighborhood, in the octant region of Fig. 3 , the area of the corresponding part of the chamfer octagon is $1 / 2 a b$, whereas the corresponding disk sector has area $\pi / 8$. Hence, forcing a zero area difference is equivalent to the constraint

$$
a b=4 / \pi
$$

For each case, the optimal $(a, b)$ were found using the optimization toolbox of MATLAB. We have experimentally found that all three approaches above yield very similar values for the optimal $(a, b)$ and the minimum MSE. We have selected approach 3, which gave $a=0.94885, b=1.34188$, and $\sigma=2.369 \%$. Choosing the zero area difference constraint is consistent with our original motivation to optimize chamfer distance transforms for multiscale morphological filtering where the chamfer balls (polygons) should best approximate the disks. Table I shows the MAE, MSE, and relative area differences between the chamfer octagon and the disk of same scale for various choices of $(a, b)$.

\section{Integer Local Distances}

In practice, for faster implementation, integer-valued local distances $A$ and $B$ are used, and the computed distance transform is divided by a normalizing constant $k$, which can be real-valued. We refer to such a metric as $(A, B) / k$. Its resulting chamfer distances are obviously the same as the ones obtained using real-valued local distances $A / k$ and $B / k$. Integer local distances can be obtained from any realvalued counterparts by multiplying the latter with $k$ and then rounding. One can arbitrarily choose the value of $k$, e.g., 100, and set the integer distances equal to $A=$ round $(k a)$ and $B=$ round $(k b)$. This may result in a suboptimum selection because the roundoff error may be large and $B / A$ may not be very close to $b / a$. We are proposing a new approach where we first select $A$ from a desired integer range, say $A<100$, such that the roundoff error $|A b / a-\operatorname{round}(A b / a)|$ is minimum. Then we set $B=\operatorname{round}(A b / a)$ and $k=A / a$. For example, 


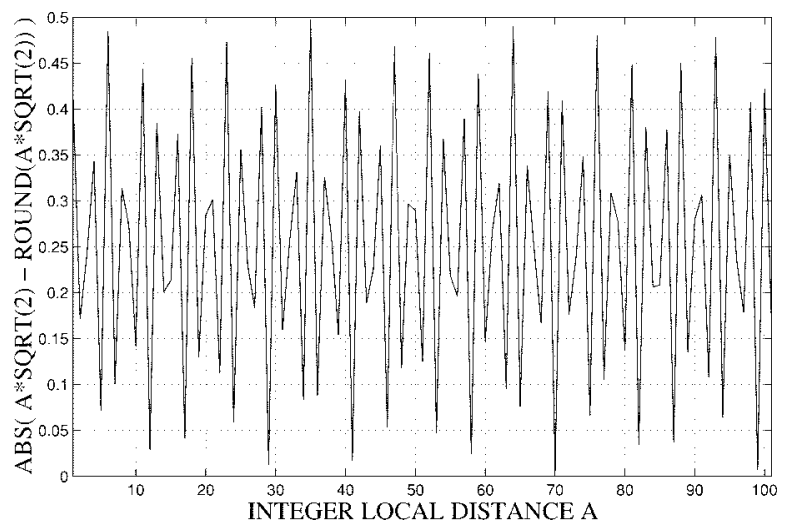

Fig. 6. Search for semioptimal integer valued local distances for minimum MAE. Along the $Y$-axis, the absolute difference between $A \sqrt{2}$ and its rounded value is plotted.

given the optimal real local distances $(a, b)$ for minimum MAE (see Table I) where $b / a=\sqrt{2}$, the search for an optimum integer $A$ yields $A=70$ (see Fig. 6), which in turn gives $B=99, k=72.77$, and MAE $=3.958 \%$. In Table I we also provide an integer approximation to the real local distances that minimize the MSE.

\section{Optimal Local Distances for $5 \times 5$ Neighborhood}

For a $5 \times 5$ neighborhood three local distances are used: the distance $a$ to the horizontal and vertical neighbors, the distance $b$ to the diagonal neighbors, and the distance $c$ to the off-diagonal knight's move neighbors (see Fig. 1). The other local distances in $5 \times 5$ neighborhood are redundant because they can be expressed in terms of $a, b$, and $c$. The chamfer polygon of size $r>0$ generated with these local distances has 16 sides. Since it is symmetric with respect to the axes and the diagonals, the error analysis can be limited to the octant shown in Fig. 7. From the geometry of Fig. 7 it can be found that the angles $\theta_{1}$ and $\theta_{2}$ are given by

$$
\begin{aligned}
& \theta_{1}=\tan ^{-1}\left(\frac{r / c}{r / a-2 r / c}\right)=\tan ^{-1}\left(\frac{a}{c-2 a}\right) \\
& \theta_{2}=\tan ^{-1}\left(\frac{r / b-r / c}{2 r / c-r / b}\right)=\tan ^{-1}\left(\frac{c-b}{2 b-c}\right) .
\end{aligned}
$$

By using the law of sines in the triangles of Fig. 7, we find that lengths of the vectors tracing the two edges $\overline{A C}$ and $\overline{C B}$ of the chamfer polygon are

$$
L(\theta)= \begin{cases}\frac{r \sin \left(\theta_{1}\right)}{a \sin \left(\theta+\theta_{1}\right)}, & \theta \in\left[0^{\circ}, 26.65^{\circ}\right] \\ \frac{r \sin \left(\theta_{2}\right)}{(c-b) \sin \left(\theta+\theta_{2}\right)}, & \theta \in\left[26.65^{\circ}, 45^{\circ}\right] .\end{cases}
$$

These expressions are then used to minimize the normalized error $E(\theta)=1-L(\theta) / r$ under various error criteria.

If exact Euclidean distances $(1, \sqrt{2}, \sqrt{5})$ are used as local distances, the corners of the unit chamfer polygon will be on the unit circle. The error plots for this chamfer metric are given in Fig. 8(a) and (b). These real-valued local distances give an MAE of $2.67 \%$ occurring at $\theta=13.28^{\circ}$. The error is always positive and consists of lobes of different heights. The MAE

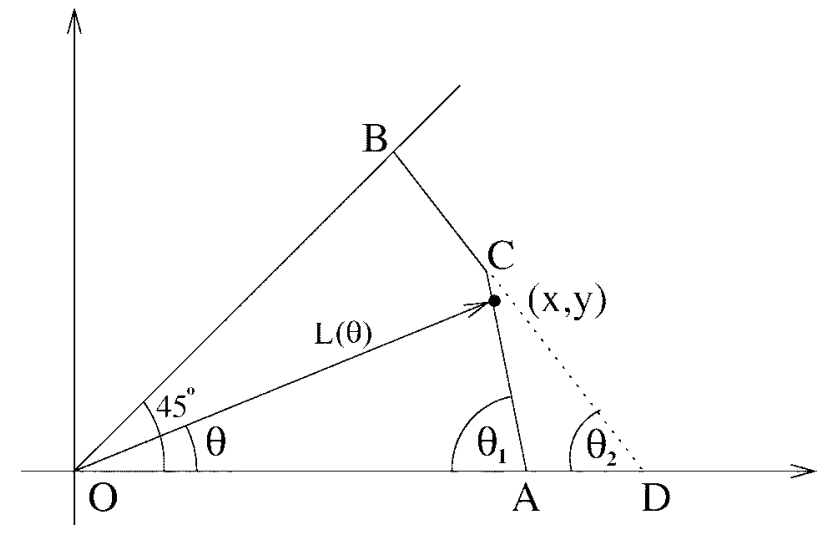

Fig. 7. Analysis of edges of the chamfer hexadecagon of size $r$ generated by the $5 \times 5$ chamfer metric $(a, b, c)$. The end points have coordinates $A=(r / a, 0), B=(r / b, r / b), C=(2 r / c, r / c)$, and $D=(r /(c-b), 0)$.

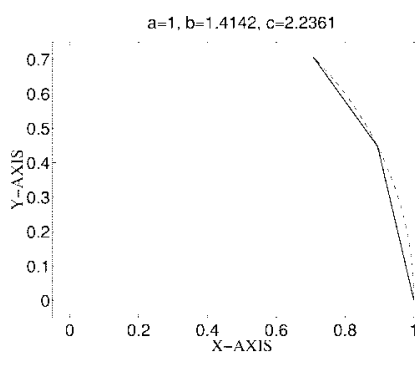

(a)

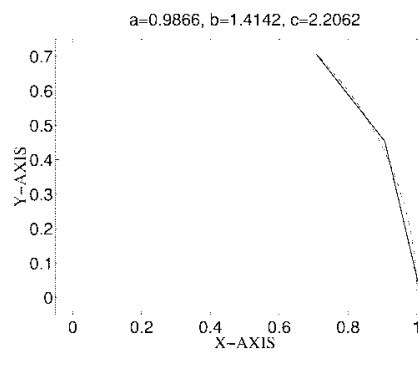

(c)

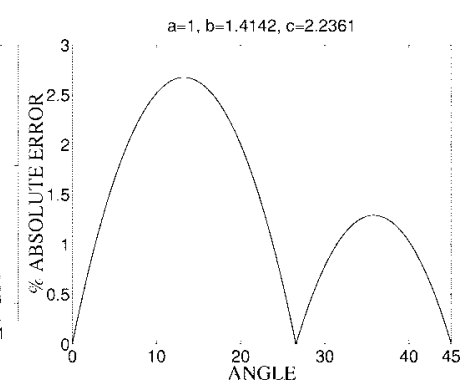

(b)

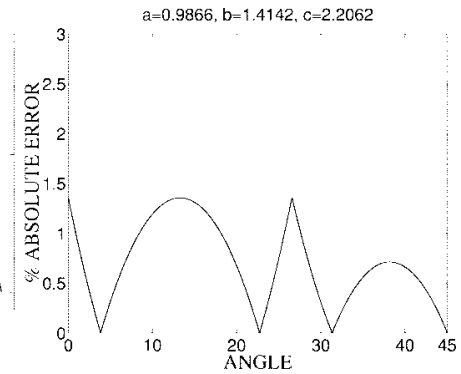

(d)
Fig. 8. Edge geometry and normalized errors for $5 \times 5$ chamfer metrics. (a), (b) Euclidean local distances $(1, \sqrt{2}, \sqrt{5})$. (c), (d) Optimal local distances $(0.9866, \sqrt{2}, 2.2062)$.

occurs at the peak of the bigger lobe corresponding to the edge $\overline{A C}$ which is controlled by the local distances $a$ and $c$. These local distances should be selected so that both corners of the edge are pushed out of the unit circle by the same amount making the triangle $O A C$ isosceles, i.e., $c=\sqrt{5} a$. In this case, $-E\left(0^{\circ}\right)=E\left(13.28^{\circ}\right)=-E\left(26.56^{\circ}\right)$. This yields the optimal value of

$$
a=\frac{1+\cos \left(13.28^{\circ}\right)}{2}=0.9866
$$

which in turn gives the optimal value of $c=\sqrt{5} a=2.2062$ and an MAE of $1.36 \%$. For minimizing the MAE, the value of $b$ is not critical because it is associated with the smaller error lobe. Once $a$ and $c$ are selected to optimally place the longer edge $\overline{A C}$ across the unit circle, $b$ can be selected in such a way that the MAE is smaller than $1.36 \%$. A possible 
TABLE II

Errors for Chamfer Distance Transforms Based on $5 \times 5$ Neighborhood

\begin{tabular}{c|r|r|r|l}
\hline$(a, b, c)$ & MAE & $\sqrt{\mathrm{MSE}}$ & Area Diff. & Comments \\
\hline$(1, \sqrt{2}, \sqrt{5})$ & $2.675 \%$ & $1.623 \%$ & $2.79 \%$ & Euclidean local distances \\
\hline$(5,7,11) / 5$ & $1.942 \%$ & $1.117 \%$ & $0.79 \%$ & A common choice \\
\hline$(0.9866, \sqrt{2}, 2.2062)$ & $1.356 \%$ & $0.804 \%$ & $0.70 \%$ & Optimal for min MAE \\
\hline$(34,48,76) / 34.45$ & $1.358 \%$ & $0.804 \%$ & $0.70 \%$ & Integer approx. for min MAE \\
\hline$(0.9802,1.4060,2.2046)$ & $2.023 \%$ & $0.708 \%$ & $0 \%$ & Optimal for min MSE with Area Diff.=0 \\
\hline$(30,43,67) / 30.57$ & $2.024 \%$ & $0.777 \%$ & $0.16 \%$ & Integer approx. for min MSE \\
\hline
\end{tabular}

such range for $b$ is

$$
1<\frac{\sqrt{2}}{b}<\frac{1}{a} \Rightarrow 1.3953<b<\sqrt{2}
$$

This procedure of finding the value of $b$ is similar to that used in [3]. Fig. 8(b) and (d) shows the error plots for the optimal chamfer metric $(0.9866, \sqrt{2}, 2.2062)$.

To find optimal $(a, b, c)$ under the MSE criterion subject to a zero area difference constraint we need to perform a numerical minimization (using MATLAB) of the expression $\int_{0}^{\pi / 4}(1-L(\theta) / r)^{2} d \theta$, where $L(\theta)$ is given by (10). The constraint that the areas of the chamfer polygon and disk parts in the octant region of Fig. 7 are equal leads to

$$
\frac{b+a}{2 a b c}=\frac{\pi}{8}
$$

The resulting local distances are given in Table II. These local distance satisfy the regularity conditions, found in [8], for the metric corresponding to the $5 \times 5$ chamfer mask.

As mentioned before, practical implementations use integervalued local distances $(A, B, C)$ and divide the final distance by a constant $k$. We denote such a chamfer metric by $(A, B, C) / k$. The best combination of integer local distances for any set of real local distances can be found by varying one of the local distances over a desired integer range and finding other integer local distances with ratios close to the ratios of the real local distances. For a $5 \times 5$ neighborhood, such optimal combinations of integer local distances and normalizing constant are given in Table II.

\section{OPTIMAL LOCAl Distances FoR \\ $7 \times 7$ AND LARGER NEIGHBORHOODS}

In the rest of this paper, chamfer metrics based on larger neighborhoods will be analyzed. Some results concerning the geometry of large chamfer masks have been obtained in [7]. In this paper, we optimize such large chamfer masks by minimizing the maximum absolute error. The optimal local distances under other error criteria can be easily found by following the analysis presented in earlier sections.

The $7 \times 7$ neighborhood has five nonredundant local distances shown in Fig. 1. The corresponding chamfer polygon has 32 sides if all of the local distances $a, b, c, d$, and $e$ are

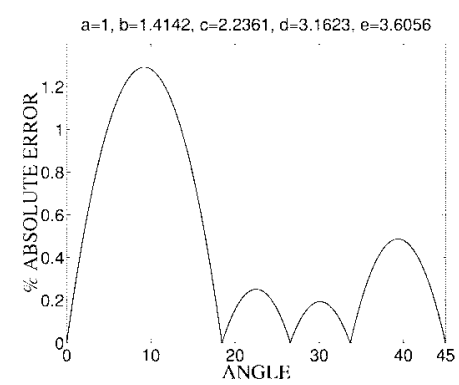

(a)

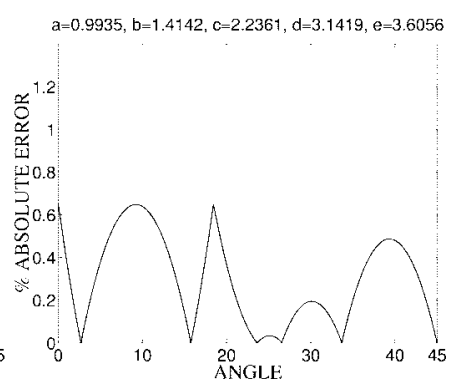

(b)
Fig. 9. Normalized errors for $7 \times 7$ chamfer metrics. (a) Euclidean local distances $(1, \sqrt{2}, \sqrt{5}, \sqrt{10}, \sqrt{13})$. (b) Optimal local distances $(0.9935, \sqrt{2}, \sqrt{5}, 3.1419, \sqrt{13})$.

used to implement the distance transform. The corners of the chamfer polygon are in the same directions as the local distance vectors. Starting from the $x$-axis and going counterclockwise, the locations of the vertices of the unit chamfer polygon are at

$$
\left(\frac{1}{a}, 0\right),\left(\frac{3}{d}, \frac{1}{d}\right),\left(\frac{2}{c}, \frac{1}{c}\right),\left(\frac{3}{e}, \frac{2}{e}\right),\left(\frac{1}{b}, \frac{1}{b}\right) .
$$

If the local distances are the exact Euclidean distances, the corners of the unit chamfer polygon will be on the unit circle; the error plots for this case are shown in Fig. 9(a) and (b). An MAE of $1.29 \%$ occurs at $\theta=9.22^{\circ}$. The error plot consists of a dominant lobe and three smaller lobes. The two middle lobes are not significant and can be combined into one without affecting the maximum error. This fusion of the lobes is achieved by eliminating the local distance corresponding to the corner between them; this should be the local distance $c$. Note that in [3] elimination of the local distance $e$ was proposed. Local distances $a$ and $d$ control the first major error lobe. Following the analysis given in the previous sections, the optimal value of $a$ is

$$
a=\frac{1+\sin \left(90^{\circ}-9.22^{\circ}\right)}{2}=0.9935
$$

which gives a maximum absolute error of $0.65 \%$. The optimal value of $d$ is $d=\sqrt{10} a=3.1419$. The values of $b, c$, and $e$ are not critical and can be selected so that the maximum error remains within bounds dictated by $a$ and $d$. One such choice could be the exact Euclidean local distances $b=\sqrt{2}, c=\sqrt{5}$, 
TABLE III

MaXimum Absolute Error for Chamfer Distance Transforms with Optimal local Distances

\begin{tabular}{c|c|c|c|c|c|c|c}
\hline Neighborhood Size & $3 \times 3$ & $5 \times 5$ & $7 \times 7$ & $9 \times 9$ & $11 \times 11$ & $13 \times 13$ & $15 \times 15$ \\
\hline Approximation Error & $3.9566 \%$ & $1.3557 \%$ & $0.6498 \%$ & $0.3760 \%$ & $0.2439 \%$ & $0.1707 \%$ & $0.1259 \%$ \\
\hline
\end{tabular}

TABLE IV

Reduction of Computation Complexity with the Use of Critical Local Distances

\begin{tabular}{c|c|c|c|c|c|c|c|c|c}
\hline Neighborhood Size & $7 \times 7$ & $9 \times 9$ & $11 \times 11$ & $13 \times 13$ & $15 \times 15$ & $17 \times 17$ & $19 \times 19$ & $21 \times 21$ & $23 \times 23$ \\
\hline$\%$ Reduction & $25 \%$ & $33 \%$ & $50 \%$ & $50 \%$ & $61 \%$ & $64 \%$ & $68 \%$ & $69 \%$ & $74 \%$ \\
\hline
\end{tabular}

and $e=\sqrt{13}$. Fig. 9(c) and (d) shows the error plots for the above optimal local distances.

In general, a smaller approximation error can be achieved by using a larger neighborhood but at the cost of a slower implementation. For a $(2 P+1) \times(2 P+1)$ neighborhood, $P=1,2,3, \cdots$, the nonredundant local distances correspond to the vectors connecting origin with the following pixels:

$$
\left\{(x, y) \in \mathbb{Z}^{2}:|x| \leq P,|y| \leq P, \quad \text { and } \operatorname{gcd}(x, y)=1\right\}
$$

where "gcd" stands for "greatest common divisor." As pointed out in [2], These local distances form the Farey sequence. If all of these local distances are used, the error plot consists of a major lobe and some minor lobes. The first lobe, associated with the edge of the chamfer polygon joining the positive $x$ axis and the corner in the direction of $\tan ^{-1}(1 / P)$, is the major lobe because this edge is the largest of all the polygon edges. It can be proved that

$$
\begin{gathered}
\tan ^{-1}\left(\frac{1}{P}\right)>\left(\tan ^{-1}\left(\frac{n}{P}\right)-\tan ^{-1}\left(\frac{n-1}{P}\right)\right), \\
n=2,3, \cdots, p
\end{gathered}
$$

because $d\left(\tan ^{-1} u\right) / d u=1 /\left(1+u^{2}\right)$ is a decreasing function for $u \geq 0$. The optimal selection of local distances, which control the vertices in the directions of positive $x$ axis and $\tan ^{-1}(1 / P)$, gives the following minimum value of normalized MAE:

$$
\min _{(a, b, c, \cdots)}\left\{\max _{\theta}|E(\theta, P)|\right\}=\frac{1-\cos \left(0.5 \tan ^{-1}(1 / P)\right)}{1+\cos \left(0.5 \tan ^{-1}(1 / P)\right)} .
$$

Table III gives these lower error bounds for different neighborhood sizes. Thus, one can get a good approximation of the Euclidean distance transform by using a suitably large neighborhood for local distance calculations, but at the expense of more computations. However, there is another possibility to increase the speed of chamfer distance transform based on large neighborhoods. As seen in the case of $7 \times 7$ neighborhood, some of the nonredundant local distances can be excluded from local distance computations without increasing the maximum absolute error. This requires fewer computations at each step of the distance propagation and hence faster implementation.

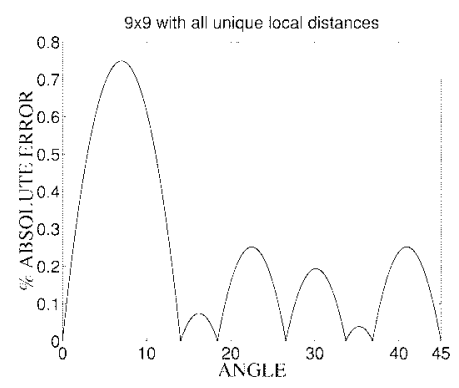

(a)

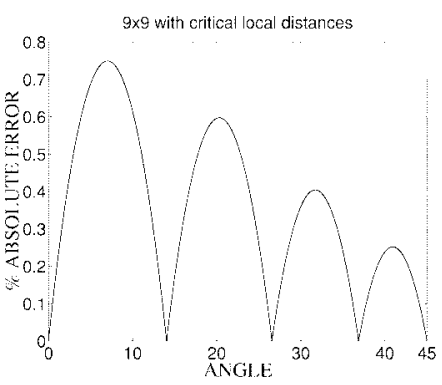

(c)

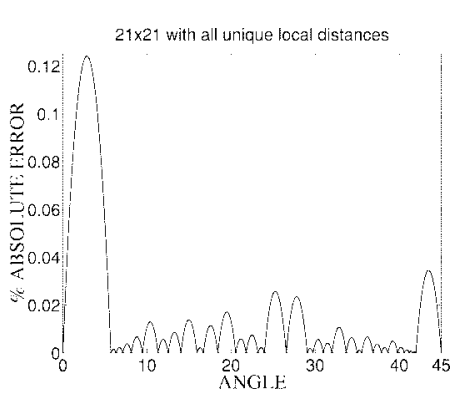

(b)

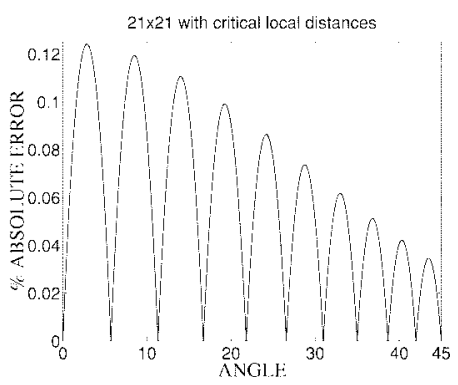

(d)
Fig. 10. Error plots for large neighborhoods when selected local distances are used. (a) For $9 \times 9$ neighborhood with all nonredundant local distances. (b) For $21 \times 21$ neighborhood with all nonredundant local distances. (c) For $9 \times$ 9 neighborhood with critical local distances. (d) For $21 \times 21$ neighborhood with critical local distances.

Fig. 10(a) and (b) shows the error plots for two large neighborhoods when all of the nonredundant local distances are used. These plots show that there are many insignificant small error lobes which can be combined without increasing the MAE. We propose that, for a neighborhood of size $(2 P+$ $1) \times(2 P+1)$, only the local distances corresponding to the following set of direction vectors should be used:

$$
\left\{(x, y) \in \mathbb{Z}^{2}: \max (|x|,|y|)=P \text {, and } \operatorname{gcd}(x, y)=1\right\} \text {. }
$$

These local distances are critical because excluding one of these can increase the maximum absolute error. Fig. 10(c) and (d) shows the error plots with critical local distances only. Using these critical local distances, only $8 P$ distance evaluations are required for each pixel. The computational reduction offered by our approach over any other approach that uses all nonredundant local distances is given in Table IV. 


\section{REFERENCES}

[1] A. Rosenfeld and J. L. Pfaltz, "Sequential operations in digital picture processing," J. ACM, vol. 13, pp. 471-496, Oct. 1966.

[2] U. Montanari, "A method for obtaining skeletons using a quasieuclidean distance," J. ACM, vol. 15, pp. 600-624, Oct. 1968.

[3] G. Borgefors, "Distance transformations in digital images," Comput. Vis., Graphics, Image Process., vol. 34, pp. 344-371, 1986.

[4] L. Vincent, "Morphological algorithms," in Mathematical Morphology in Image Processing, E. Dougherty, Ed. New York: Marcel Dekker, 1992, pp. 133-134.

[5] P. Maragos, "Differential morphology and image processing," IEEE Trans. Image Processing, vol. 5, pp. 922-937, June 1996.

[6] H. Barrow, J. Tenenbaum, R. Boles, and H. Wolf, "Parametric correspondence and chamfer matching: Two new techniques for image matching," in Proc. 5th Int. Joint Conf. Artificial Intelligence, Cambridge, MA, 1977, pp. 659-663.

[7] E. Thiel and A. Montanvert, "Chamfer masks: Discrete distance functions, geometric properties and optimization," in Proc. 11th Int. Conf. Pattern Recognition, The Hague, The Netherlands, Apr. 1992, pp. 244-247.

[8] C. Kiselman, "Regularity properties of distance transformations in image analysis," Comput. Vis. Image Understand., vol. 64, pp. 390-398, Nov. 1988.

[9] A. Vossepoel, "A note on distance transformations in digital images," Comput. Vis., Graphics, Image Process., vol. 43, pp. 88-97, 1988.

[10] A. Beckers and A. Smeulders, "A comment on "A note on distance transformations in digital images'," Comput. Vis., Graphics, Image Process., vol. 47, pp. 89-91, 1989.

[11] G. Borgefors, "Another comment on 'a note on distance transformations in digital images", Comput. Vis., Graphics, Image Process.: Image Understand., vol. 54, pp. 301-306, Sept. 1991.

[12] L. Dorst and A. Smeulders, "Best linear unbiased estimators for properties of digitized straight lines," IEEE Trans. Pattern Anal. Mach. Intell., vol. PAMI-8, pp. 276-282, Mar. 1986.

[13] B. Verwer, "Local distances for distance transformations in two and three dimensions," Pattern Recognit. Lett., vol. 12, pp. 671-682, 1991.

[14] M. Yamashita, "Distance defined by neighborhood sequences," Pattern Recognit., vol. 19, pp. 237-246, 1986.

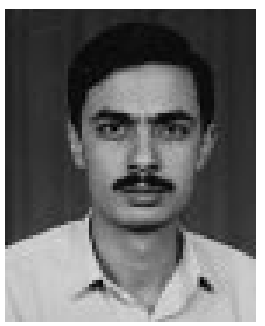

Muhammad Akmal Butt (S'96) received the B.S.E.E. degree from the University of Engineering and Technology, Lahore, Pakistan, in 1990, and the M.S.E.E. degree from the University of Wisconsin, Madison, in 1993. He has been with the Georgia Institute of Technology, Atlanta, since 1993, where he is currently a Ph.D. candidate in the School of Electrical and Computer Engineering.

His main research interests are nonlinear image processing and computer vision.

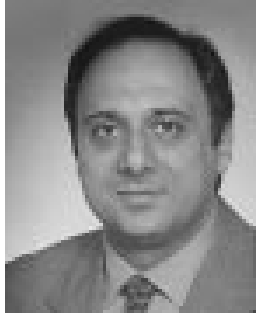

Petros Maragos (S'81-M'85-SM'91-F'95) received the Diploma degree in electrical engineering from the National Technical University of Athens, Greece, in 1980, and the M.S.E.E. and Ph.D. degrees from the Georgia Institute of Technology, Atlanta, in 1982 and 1985, respectively.

In 1985, he joined the faculty of the Division of Applied Sciences at Harvard University, Cambridge, MA, where he worked as Assistant (1985-1989) and Associate Professor (1989-1993) of electrical engineering, affiliated with the interdisciplinary Harvard Robotics Laboratory. He has also been a consultant to several industry research groups including Xerox's research on document image analysis. In June 1993, he joined the faculty of Georgia Tech, where he is currently an Associate Professor of electrical and computer engineering. During parts of 1995-1997, he has been on academic leave working as a Visiting Professor at the National Technical University of Athens and as a Senior Researcher at the Institute for Language and Speech Processing, Athens.

Dr. Maragos has served as Associate Editor for the IEEE TRAnsactions on Signal Processing and as Guest Editor for the IEEE Transactions ON IMAge PRoCEssing; General Chairman for the 1992 SPIE Conference on Visual Communications and Image Processing, and Co-Chairman for the 1996 International Symposium on Mathematical Morphology; Member of two IEEE DSP Committees; and President of the International Society for Mathematical Morphology. He has received several awards, including a 1987 NSF Presidential Young Investigator Award; the 1988 IEEE Signal Processing Society's Paper Award for the paper "Morphological filters"; the 1994 IEEE Signal Processing Society's Senior Award; the 1995 IEEE Baker Award for the paper "Energy separation in signal modulations with application to speech analysis" (co-recipient); and the 1996 Pattern Recognition Society's Honorable Mention Award for the paper "Min-max classifiers" (co-recipient). 